\title{
DEC1: a potential biomarker of malignant transformation in oral leukoplakia
}

\author{
Ting $M \mathrm{O}^{(a)}$ (iD \\ Haofeng XIONG(b) iD \\ Xin $\mathrm{HU}^{(a)}$ \\ Yue HU(a) ID \\ Can WANG(a) iD \\ Liudi YANG(a) ID \\ Danni HUANG(a) iD \\ Kun XIA ${ }^{(\mathbf{b})}$ \\ Tong SU(a) \\ (a)Central South University, Xiangya Hospital, \\ Center of Stomatology, Changsha, Hunan, \\ China. \\ (b) Central South University, Center for \\ Medical Genetics, School of Life Sciences, \\ Changsha, Hunan, China.
}

Declaration of Interests: The authors certify that they have no commercial or associative interest that represents a conflict of interest in connection with the manuscript.

Corresponding Author:

Tong Su

E-mail: sutong@csu.edu.cn

https://doi.org/10.1590/1807-3107bor-2020.vol34.0052

Submitted: October 7, 2019

Accepted for publication: March 16, 2020

Last revision: April 4, 2020

\begin{abstract}
The purpose of this study was to analyze the differential expression of DEC1 in oral normal mucosa (NM), oral leukoplakia (OLK) and oral squamous cell carcinoma (OSCC). Surgically excised specimens from patients with OLK $(n=47)$, OSCC $(n=30)$ and oral normal mucosa $(n=11)$ were immunostained for DEC1. The expression of DEC1 protein was evaluated, and its association with the clinicopathological features was analyzed. The expression of DEC1 in NM, OLK and OSCC tissues increased in turn, and significant differences were observed among the groups $(P<0.0001)$. In terms of the association between DEC1 expression and epithelial dysplasia, DEC1 expression was lower in hyperkeratosis without dysplasia (H-OLK) than in OLK with moderate to severe dysplasia (S-OLK), and these differences were significant $(p<0.05)$. The expression of DEC1 in OSCC with OLK was significantly higher than that in OSCC without OLK $(\mathrm{p}<0.01)$. Therefore, DEC1 could be a potential biomarker of malignant transformation in the carcinogenesis of OSCC, which may provide a new research direction for the transformation of oral potentially malignant disorders (OPMDs) into OSCC.
\end{abstract}

Keywords: Leukoplakia, Oral; Mouth Neoplasms.

\section{Introduction}

Oral leukoplakia (OLK) is a white plaque in the oral cavity that cannot be wiped away and cannot be diagnosed as any other disease. ${ }^{1}$ It is well known that OLK is the most common form of oral potentially malignant disorders (OPMDs). ${ }^{2}$ According to its pathological features, OLK can be divided into epithelial hyperplasia or hyperkeratosis, with or without epithelial dysplasia. ${ }^{3}$ The probability of malignant transformation of OLK was $0.13-17.5 \%$ in one study, which was proportional to the degree of epithelial cell dysplasia. ${ }^{4}$ Oral epithelial cell dysplasia (OED) had malignant conversion rates of $6.6 \%-36.4 \%$, which may increase the risk of OLK malignant transformation. A malignant conversion rate of less than $5 \%$ was associated with mild OED, while rates of $3-15 \%$ and $7-50 \%$ were associated with moderate and severe OED, respectively. ${ }^{5}$

Oral squamous cell carcinoma (OSCC), the most common head and neck malignancy, is the worst end point of OLK development. ${ }^{6}$ OSCC is a preventable disease, as approximately $62 \%$ of cases are transformed from 
OPMDs. ${ }^{7}$ Thus, more attention should be focused on the search for a reliable biomarker for the early detection of OSCC.

According to early research by our team, DEC1 expression was found to be correlated with the incidence and prognosis of OSCC. ${ }^{8}$ Human differentiated embryonic chondrocyte-expressed gene 1 (DEC1, also known as Bhlhe40/Bhlhb2/ Stra13/Sharp2), a member of the basic helix-loophelix (bHLH) transcription factor family, plays an important role in the development of chondrocytes and in the regulation of circadian rhythms. ${ }^{9}$ In previous studies, DEC1 was reported to be a useful marker in detecting the occurrence of tumors and evaluating tumor prognosis. ${ }^{10,11}$ In gastric cancer, DEC1 was shown to be highly expressed and positively correlated with the expression of KI67.10 DEC1 was further demonstrated to be essential for gastric cancer cell proliferation and for the promotion of tumor invasiveness in gastric cancer. ${ }^{10}$ Similarly, in studies on hepatocellular carcinoma and pancreatic ductal adenocarcinoma, DEC1 is often regarded as a potential biological marker for the occurrence and prognosis of cancer. ${ }^{11}$ Therefore, in this study, we focused on the differential expression of DEC1 protein during the evolution of normal oral mucosa to OLK and then to OSCC.

\section{Methodology}

\section{Patients and clinical specimens}

Ethical approval for this study was obtained from the Medical Ethics Committee of Xiangya Hospital, Central South University. We reviewed all biopsies that were histologically diagnosed as OLK or OSCC at two institutions: the Department of Pathology, Xiangya Hospital (from May 2014 to May 2018), Central South University and the Department of Oral Pathology, Xiangya Stomatological Hospital, Central South University (from May 2014 to May 2018). We have read the Declaration of Helsinki and have followed the guidelines in this research. All clinicopathological information such as gender, age, site of lesion, size and pathological findings was obtained from medical records (Table 1).
Table 1. Clinicopathological informations.

\begin{tabular}{|c|c|c|}
\hline Clinicopathological features & $\begin{array}{c}\text { Number of } \\
\text { samples }\end{array}$ & $\%$ \\
\hline \multicolumn{3}{|l|}{ Age (years) } \\
\hline$>50$ & 36 & 46.8 \\
\hline$\leq 50$ & 41 & 53.2 \\
\hline \multicolumn{3}{|l|}{ Gender } \\
\hline Male & 71 & 92.2 \\
\hline Famale & 6 & 7.8 \\
\hline \multicolumn{3}{|l|}{ Site } \\
\hline Tongue & 56 & 72.7 \\
\hline Buccal & 19 & 24.7 \\
\hline Gingiva & 2 & 2.6 \\
\hline \multicolumn{3}{|l|}{ Histopathological grading } \\
\hline Hyperkeratosis without dysplasia & 12 & 15.6 \\
\hline Mild dysplasia & 21 & 27.3 \\
\hline Moderate and severe dysplasia & 14 & 18.2 \\
\hline OSCC risen from oral leukoplakia & 12 & 15.6 \\
\hline Oscc & 18 & 23.4 \\
\hline \multicolumn{3}{|l|}{ Habits } \\
\hline \multicolumn{3}{|l|}{ Tobacco } \\
\hline Alcohol & \multicolumn{2}{|l|}{ NA } \\
\hline Areca nut chewing & & \\
\hline
\end{tabular}

Samples used in this study consisted of surgically excised specimens from 77 patients, among which 47 were diagnosed as OLK and 30 were diagnosed as OSCC. A previously reported oral epithelial dysplasia scoring system was used to grade the OED in this study. ${ }^{12}$ Oral normal mucosa (NM) tissues were obtained from eleven healthy volunteers. All the normal mucosa specimens were derived from the extra normal oral mucosa, which was removed during surgery for maxillofacial trauma or cleft lip and palate. Histopathological diagnosis was confirmed by two experienced pathologists by reviewing the original histological sections stained with hematoxylin and eosin.

\section{Immunohistochemistry}

After the specimens were resected, they were fixed in $10 \%$ formalin, embedded in paraffin, sectioned 
into $4 \mu \mathrm{m}$ slices and placed on slides. The slides were deparaffinized for 60 minutes in a temperature chamber at $65^{\circ} \mathrm{C}$. Following deparaffinization by immersion in xylene, the sections were immersed in alcohol then washed in distilled water. Antigen retrieval was performed as previously reported. ${ }^{13}$ Then, the slices were washed three times in phosphate buffer for 5 minutes each time.

Endogenous peroxidase activity was blocked by incubating the slices with hydrogen peroxide for 20 minutes at room temperature. The sections were then incubated with a rabbit monoclonal antibody against human DEC1 (1:1000; ab70723; Abcam, USA) at $4^{\circ} \mathrm{C}$ overnight. Histostain ${ }^{\mathrm{TM}}$ SP Kits (Zsbio, Beijing, China) were used in this experiment. After a rewarming step at $37^{\circ} \mathrm{C}$, the sections were washed with PBS, incubated with goat anti-rabbit secondary antibody (Zsbio, Beijing, China) and horseradish peroxidase (HRP, Dako, Denmark) for 20 minutes at $37^{\circ} \mathrm{C}$ and then stained with 3, 3'-diaminobenzidine (DAB) for 2 minutes and counterstained with hematoxylin. After that, the sections were dehydrated and sealed for observation under a microscope.

The expression of DEC1 was reflected by the mean of the integral optical density (MOD) value, as previously reported. ${ }^{13}$ MOD values greater than 5 were defined as high expression, while MOD values less than or equal to 5 were defined as low expression. The obtained sections were observed under by microscopy. The imaging system included a Leica DFC 420 CCD camera connected to a Leica DM IRE2 microscope (Leica Microsystems Imaging Solutions, Cambridge, UK). Using Leica Qwin Plus V3 software, five representative views were selected under 400x magnification and were saved. The MOD of each image was counted and measured by Image-Pro Plus V6.0 software (Media Cybernetics, Bethesda, MD, USA). Two researchers analyzed the expression and analyzed the clinical data in a blinded manner.

\section{Statistical analysis}

All statistical analyses were performed using Graphpad Prism v6.0 software (GraphPad Software, La Jolla, USA) and SPSS 24.0 (SPSS, Inc., Chicago,
USA). Group comparisons were analyzed using oneway analysis of variance (ANOVA). Nonparametric data comparisons between groups were made with the Mann-Whitney test, while unpaired t-tests were used for parametric data. The association between clinicopathological features and the expression of DEC1 was verified by Chi-square test. The expression in each group was reflected by the mean \pm SEM. The level of statistical significance was accepted at $\mathrm{p}<0.05$.

\section{Results}

\section{Patient information}

Samples were roughly divided into three main groups according to their clinical and pathological diagnosis: the NM group $(\mathrm{n}=11)$, the OLK group $(n=47)$ (after OLK excision, patients in the OLK group were observed for approximately 1-2 years during which no malignant changes were observed) and the OSCC group ( $n=30$, no lymph node metastasis). According to the different histological manifestations and the OED classification system, the OLK group could be further subdivided into the hyperkeratosis without dysplasia subgroup (H-OLK, $\mathrm{n}=12)$, the mild dysplasia subgroup (M-OLK, $\mathrm{n}=22$ ) and the moderate to severe dysplasia subgroup (S-OLK, $n=13$ ). The OSCC group included OSCC arising from OLK (OLK-OSCC; OLK with carcinogenesis at focal sites was included in the OLK-OSCC subgroup, $n=12$ ) and the other OSCC (OSCC not arising from OLK) subgroup $(\mathrm{n}=18)$ (Table 2).

\section{Expression level of DEC1 protein in the three main groups}

Differences in the expression of DEC1 were observed among the three main groups $(p<0.0001)$. DEC1 immunohistochemical staining was observed in both the cytoplasm and nucleus but was predominantly seen in the basal and stratum spinosum layers.

In the OLK group (MOD = $15.14 \pm 1.930)$, the expression of DEC1 in the epithelial layer was significantly higher than that in the NM (MOD = $0.1648 \pm 0.09857)$ but was lower than that in the OSCC group $(\mathrm{MOD}=23.52 \pm 3.051)(\mathrm{p}<0.0001$, Figure 1$)$. 
Table 2. The samples were grouped according to histopathological grading and the specific clinical details after grouping.

\begin{tabular}{|c|c|c|c|c|c|c|c|}
\hline Clinicopathological features & $\begin{array}{c}\text { Hyperkeratosis } \\
\text { without dysplasia }\end{array}$ & $\begin{array}{c}\text { Mild } \\
\text { dysplasia }\end{array}$ & $\begin{array}{l}\text { Moderate and } \\
\text { severe dysplasia }\end{array}$ & $\begin{array}{l}\text { OSCC risen from } \\
\text { oral leukoplakia }\end{array}$ & OSCC & Total & Control \\
\hline \multicolumn{8}{|l|}{ Gender } \\
\hline Male & 12 & 19 & 13 & 11 & 16 & 71 & \multirow{8}{*}{ NA } \\
\hline Famale & 0 & 2 & 1 & 1 & 2 & 6 & \\
\hline \multicolumn{8}{|l|}{ Age(years) } \\
\hline Age range & $35-67$ & $29-64$ & $27-85$ & $36-69$ & $40-67$ & $27-85$ & \\
\hline Mean age & 49.00 & 46.48 & 51.64 & 50 & 54.11 & 50.14 & \\
\hline \multicolumn{7}{|l|}{ Anatomic site } & \\
\hline Buccal & 2 & 8 & 3 & 0 & 6 & 19 & \\
\hline Tongue & 10 & 13 & 11 & 12 & 10 & 56 & \\
\hline Gingiva & 0 & 0 & 0 & 0 & 2 & 2 & \\
\hline Numbers & 12 & 21 & 14 & 12 & 18 & 77 & 11 \\
\hline
\end{tabular}

NA: not available; OSCC: oral squamous carcinoma; N: no.
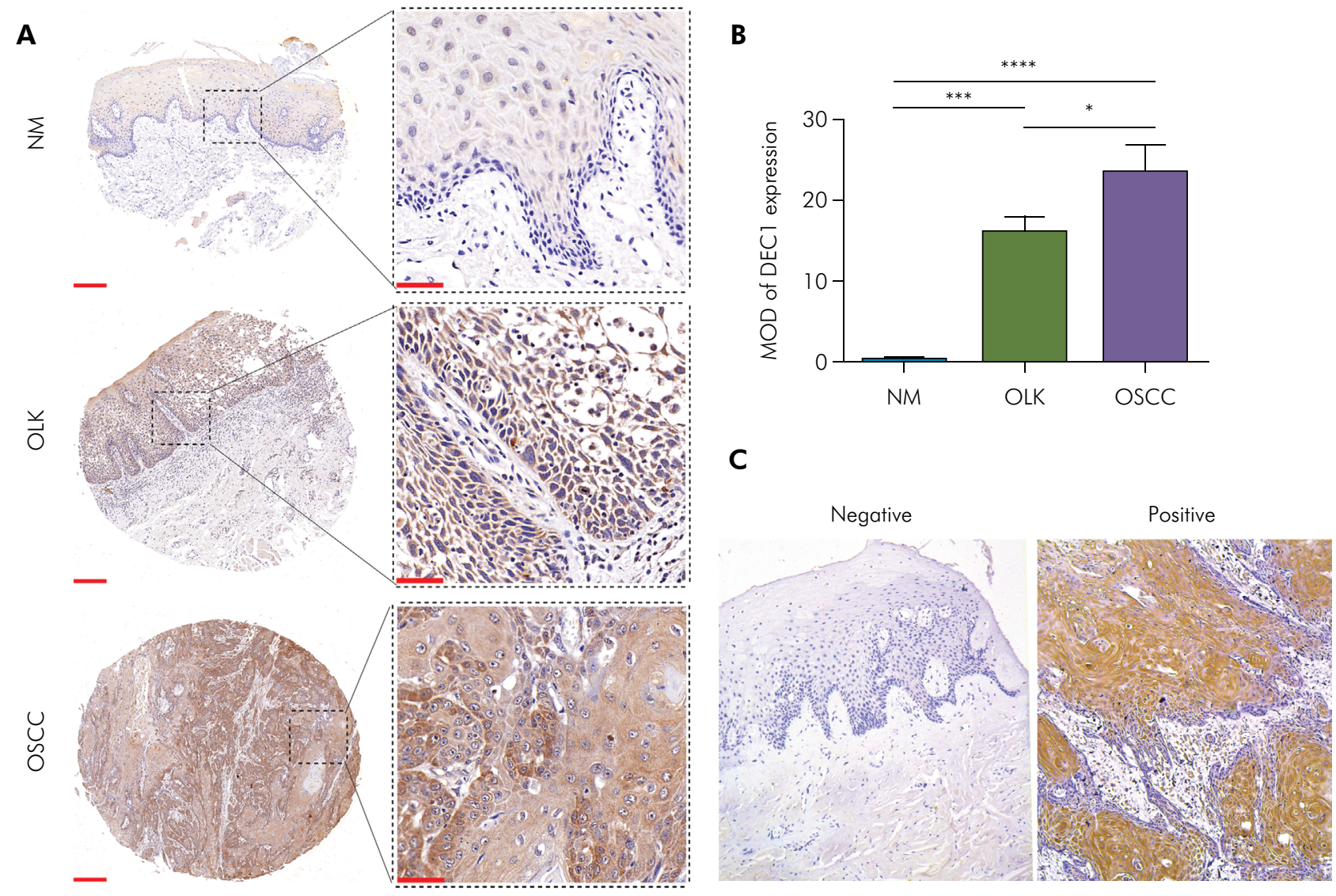

Figure 1. Immunohistochemical expression of DEC 1 in oral normal mucosa (NM), oral leukoplakia (OLK) and oral squamous cell carcinoma (OSCC): (A) DEC1 immunohistochemical staining was observed in both the cytoplasm and nucleus and was successively increased in NM, OLK and OSCC. Scale bar (left side) $=200 \mu \mathrm{m}$, scale bar (right side) $=50 \mu \mathrm{m}$. (B) The MOD of DEC 1 expression increased in turn, and the difference was statistically significant. The difference between NM and OSCC was the most obvious, followed by differences between NM and OLK, and OLK and OSCC. *indicates $\mathrm{p}<0.05,{ }^{* * *}$ indicates $\mathrm{p}<0.001,{ }^{* * * *}$ indicates $p<0.0001$, the bar on the column refers to SEM: Structural Equation Modeling. 


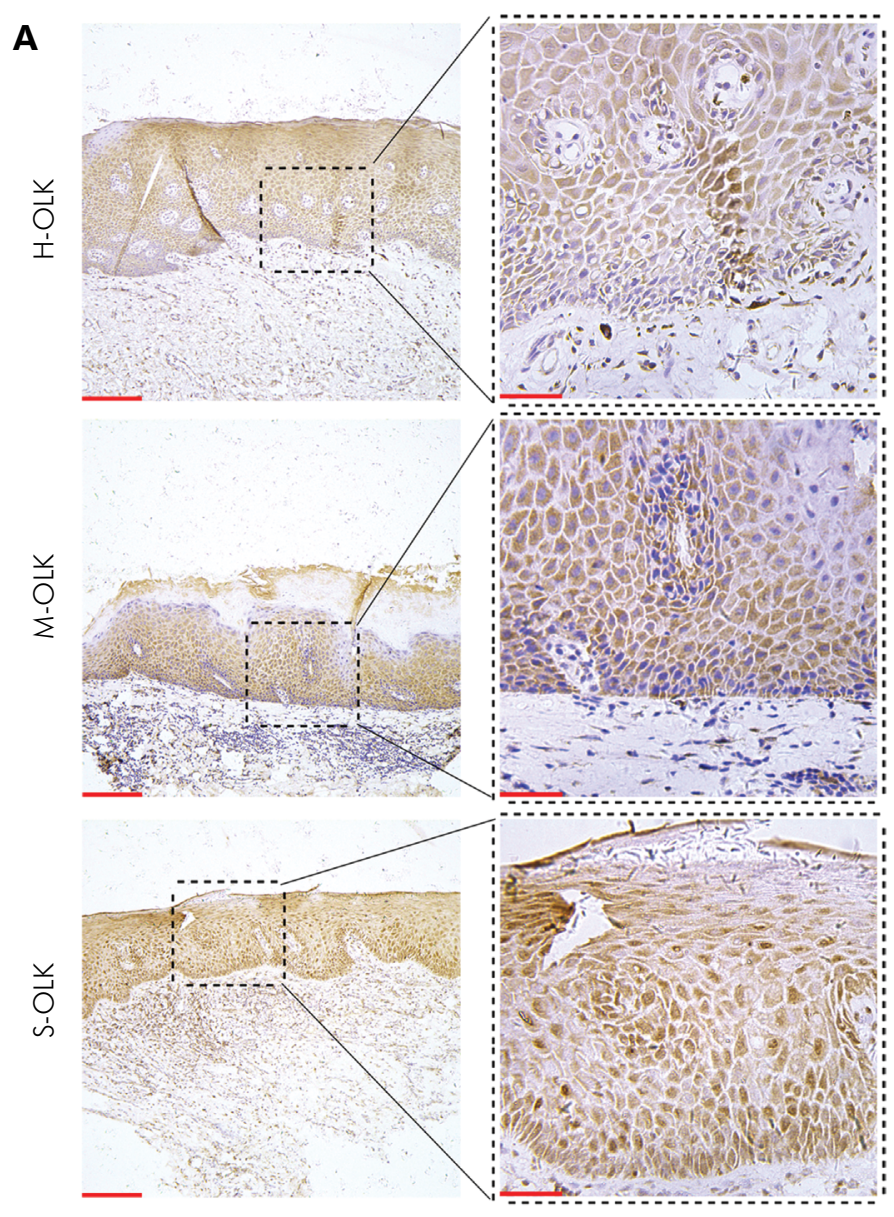

B

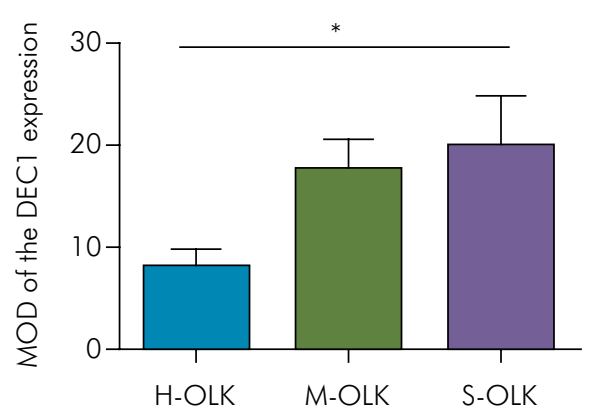

Figure 2. The connection between te expression of DEC1 and the degree of epithelial dysplasia with oral leukoplakia (OLK): (A) Immunohistochemical staining for DEC1 in the OLK group. Scale bar (left side) $=100 \mu \mathrm{m}$, scale bar (right side) $=25 \mu \mathrm{m}$. (B) In the OLK group, the expression of DEC1 in the mild dysplasia subgroup (M-OLK) was higher than that in the hyperkeratosis without dysplasia subgroup $(\mathrm{H}-\mathrm{OLK})$ and lower than that in the moderate to severe dysplasia subgroup (S-OLK). The difference between $\mathrm{H}$-OLK and S-OLK was statistically significant. ${ }^{*}$ indicates $\mathrm{p}<0.05$, the bar on the column refers to SEM: Structural Equation Modeling.

\section{Expression level of DEC1 protein in OLK tissues}

In the OLK group, the expression of DEC1 was lowest in H-OLK $(\mathrm{MOD}=8.561 \pm 1.935)$, slightly higher in M-OLK (MOD $=16.97 \pm 2.906)$ and was highest in $\mathrm{S}-\mathrm{OLK}(\mathrm{MOD}=17.43 \pm 4.140)$, but the differences were not statistically significant $(\mathrm{p}=0.0628)$. The difference in DEC1 expression between the hyperkeratosis without dysplasia subgroup and the moderate to severe dysplasia subgroup was significant $(p<0.05$, Figure 2).

\section{Expression level of DEC1 protein in OSCC tissues}

In the OSCC group, the expression of DEC1 in the OLK-OSCC subgroup (MOD = $33.52 \pm 5.406$ ) was slightly higher than that in the OSCC subgroup $(\mathrm{MOD}=16.99 \pm 3.045)$. The difference was statistically significant $(p=0.0077$, Figure 3$)$.

\section{Association between the expression level of DEC1 protein and clinicopathologic features}

Differences in DEC1 expression between groups according to age, sex and anatomical location were not observed ( $p>0.05$, Table 3.). 
A

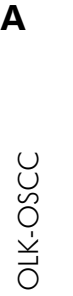

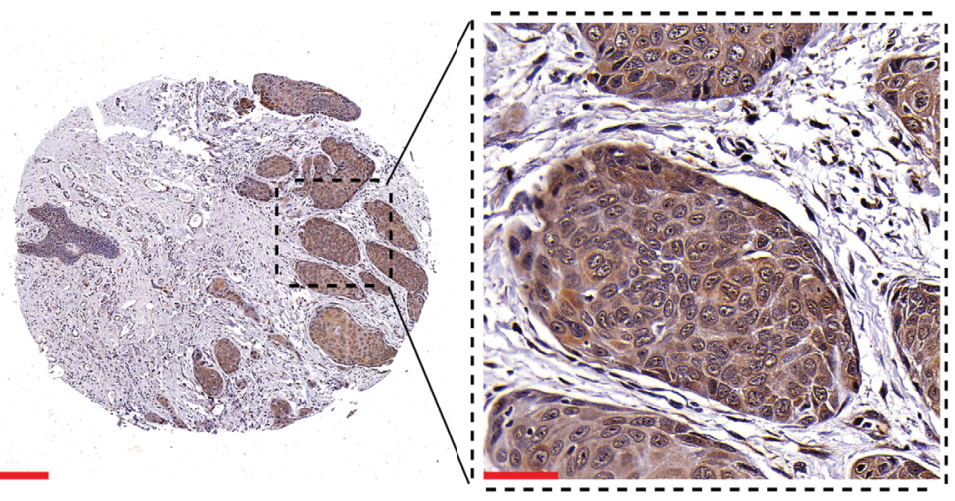

B

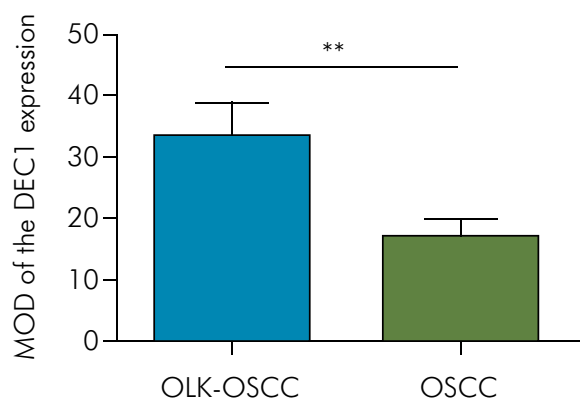

U

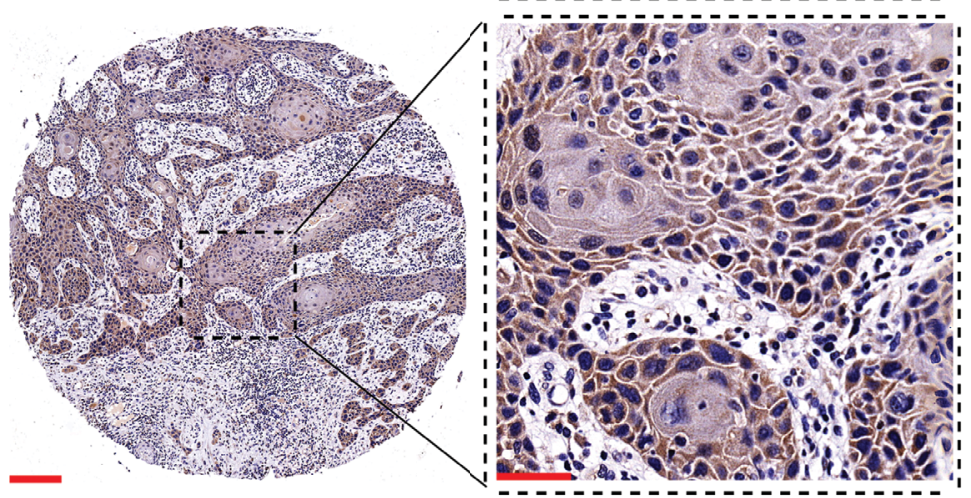

Figure 3. Differential expression of $D E C 1$ between oral squamous cell carcinoma (OSCC) that originated from oral leukoplakia (OLK) and other OSCC: (A) Immunohistochemical staining for DEC1 in OSCC and OLK-OSCC. Scale bar (left side) $=200 \mu \mathrm{m}$, scale bar (right side) $=50 \mu \mathrm{m}$. (B) The expression of DEC1 in the OSCC group in OSCC that originated from OLK was higher than that in OSCC that did not, and the difference was statistically significant. ${ }^{*}$ indicates $p<0.01$, the bar on the column refers to SEM: Structural Equation Modeling.

Table 3. Analysis of association between clinicopathological information and DEC1 expression.

\begin{tabular}{|c|c|c|c|c|c|c|c|}
\hline Clinicopathological features & $\begin{array}{l}\text { High expression } \\
\text { of } \mathrm{DEC} 1\end{array}$ & $\%$ & $\begin{array}{l}\text { Low expression } \\
\text { of } \mathrm{DECl}\end{array}$ & $\%$ & Total & Control & $\mathrm{p}$-value \\
\hline Age (years) & & & & & & & $>0.05$ \\
\hline$>50$ & 28 & 22 & 8 & 78 & 36 & \multirow{9}{*}{ NA } & \\
\hline$\leq 50$ & 32 & 22 & 9 & 78 & 41 & & \\
\hline Gender & & & & & & & $>0.05$ \\
\hline Male & 54 & 76.1 & 17 & 23.9 & 71 & & \\
\hline Famale & 6 & 100 & 0 & 0 & 6 & & \\
\hline Site & & & & & & & $>0.05$ \\
\hline Tongue & 42 & 75 & 14 & 25 & 56 & & \\
\hline Buccal & 16 & 84.2 & 3 & 15.8 & 19 & & \\
\hline Gingiva & 2 & 100 & 0 & 0 & 2 & & \\
\hline Histopathological grading & & & & & & & $>0.05$ \\
\hline Hyperkeratosis without dysplasia & 8 & 66.7 & 4 & 33.3 & 12 & & \\
\hline Mild dysplasia & 17 & 81 & 4 & 19 & 21 & & \\
\hline Moderate and severe dysplasia & 10 & 71.4 & 4 & 28.6 & 14 & 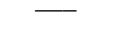 & \\
\hline OSCC risen from oral leukoplakia & 12 & 100 & 0 & 0 & 12 & & \\
\hline Oscc & 13 & 72.2 & 5 & 27.8 & 18 & & \\
\hline
\end{tabular}

NA: not avaliable; N: no; OSCC: Oral squamous cell carcinoma; High expression means MOD > 5; Low expression means MOD $\leq 5$. 


\section{Discussion}

A systematic review and meta-analysis performed by Tan $\mathrm{N}$ et al. revealed that the prevalence of OLK was $4.11 \%$ and that the malignant transformation rate was $0.13-17.5 \% .^{13}$ However, because the risk of malignant transformation of OLK is difficult to assess, it is impossible to completely prevent malignant transformation by early ablative surgery. Molecular diagnosis has been widely used in the study of various diseases to assess disease risk, presence and therapeutic efficacy. Accordingly, research on potential biomarkers that can detect the risk of malignant transformation for OLK is meaningful. In previous studies, EZH2 was reported to be a predictor of oral cancer development from OLK; E-cadherin and tenascin are also potential biomarkers of malignant transformation in oral leukoplakia, and the expression of Podoplanin in oral leukoplakia was reported to be significantly associated with the degree of epithelial atypical hyperplasia. ${ }^{14,15,16,7}$

A few studies have been performed to determine the role of DEC1 in the malignant transformation of potentially malignant disorders in addition to OPMDs. In the present study, we observed that the expression of DEC1 increased gradually in normal mucosa, OLK and OSCC, while no statistically significant difference was observed regarding age, gender, and the site of occurrence.

Carcinogenesis of oral leukoplakia is often accompanied by some clinical manifestations, histopathological changes, gene mutations and changes in the expression levels of certain genes, which can predict the risk of malignant transformation to some extent (currently, the grade of oral epithelial dysplasia is the standard by which malignant transformation is predicted). Previous studies have shown that highrisk clinical factors for oral leukoplakia canceration include: female sex, long-standing cases, lack of smoking habit, high-risk sites (body of the tongue, floor of the mouth, corner of the mouth), nonhomogenous variant, lesions greater than $>200 \mathrm{~mm}^{2}$ in size, and the presence of dysplasia. ${ }^{18}$ Additionally, P53 mutations, cell motility, and hypoxia in the oral environment are important risk factors for malignant transformation and can be detected early in the development of
OSCC. ${ }^{15,16,19}$ DEC1, as a transcription factor, can regulate epithelial-to-mesenchymal transition and the response to hypoxia in addition to its ability to regulate chondrocytes and circadian rhythms. ${ }^{20,21}$ Furthermore, preliminary research by our team discovered that expression levels of DEC1 were associated with different prognoses of OSCC. ${ }^{8}$ Taken together, DEC1 is a promising molecular biomarker of carcinogenesis, which may explain the differences in its expression among oral normal mucosa, OLK and OSCC.

In our study, the expression of DEC1 was not significantly associated with the degree of epithelial dysplasia, although it was slightly increased with the degree of epithelial dysplasia in OLK. However, the difference between H-OLK and S-OLK was statistically significant. Two possible reasons may explain this phenomenon. One is that DEC1 may be involved in the regulation of cell senescence, which leads to canceration, while the degree of epithelial dysplasia is a pathological classification of leukoplakia from the perspective of cell morphology. ${ }^{22}$ Therefore, we propose the hypothesis that DEC1 promotes canceration by regulating the aging process of cells rather than by changing the degree of atypical hyperplasia, which may require more evidence from further studies. The other is that the small sample size in each subgroup may have led to bias, and thus more reliable results should be anticipated in largescale investigations.

Additionally, we observed that the expression of DEC1 in OSCC with OLK was significantly higher than that in OSCC without OLK. Moreover, the pathological diagnosis of specimens from the OLKOSCC subgroup was incipient carcinoma. Therefore, this difference may be due to the finding that DEC1 may show an upward trend during the initiation of a carcinoma and a downward trend during the development of carcinoma. Previous studies showed that the expression of DEC1 in poorly differentiated OSCC was lower than that in highly differentiated OSCC. ${ }^{23}$ Paradoxically, in a study on the association between the prognosis of OSCC and DEC1 expression, the expression level of DEC1 in OSCC was significantly higher than that in the normal mucosa. ${ }^{8}$ Thus, the elucidation of specific signaling pathways related 
to DEC1 expression and cancer prognosis requires further study.

\section{CONCLUSION}

In summary, the DEC1 protein expression was sequentially increased in NM, OLK and OSCC, and thus this protein may be used as a novel biomarker to detect the risk of malignant transformation from OLK to OSCC.

\section{Acknowledgements}

The author is grateful for research support from Professor Zhigang Yao, the Department of Oral Pathology, Xiangya Stomatological Hospital, Central South University (CSU), China. This work was supported by the National Natural Science Foundation (81873717), the Natural Science Foundation of Hunan Province (2019JJ40508), and the Graduate Students Independently Explore Innovative Projects of Central South University (2019zzts787), China.

\section{References}

1. Chen H, Liu X, Jin Z, Gou C, Liang M, Cui L, et al. A three miRNAs signature for predicting the transformation of oral leukoplakia to oral squamous cell carcinoma. Am J Cancer Res. 2018 Aug;8(8):1403-13.

2. Mello FW, Miguel AFP. Prevalence of oral potentially malignant disorders: a systematic review and meta-analysis. J Oral Pathol Med. 2018 Aug;47(7):633-40. https://doi.org/10.1111/jop.12726

3. Wu W, Wang Z, Zhou Z. Risk factors associated with malignant transformation in patients with oral leukoplakia in a Chinese population: a retrospective study. J Oral Maxillofac Surg. 2019 Dec;77(12):2483-93. https://doi.org/10.1016/i.joms.2019.08.002

4. Dong $Y, C$ Chen $Y$, Tao $Y$, Hao Y, Jiang L, Dan H, et al. Malignant transformation of oral leukoplakia treated with carbon dioxide laser: a meta-analysis. Lasers Med Sci. 2019 Feb;34(1):209-21. https://doi.org/10.1007/s10103-018-2674-7

5. Field EA, McCarthy CE, Ho MW, Rajlawat BP, Holt D, Rogers SN, et al. The management of oral epithelial dysplasia: the Liverpool algorithm. Oral Oncol. 2015 Oct;51(10):883-7. https://doi.org/10.1016/j.oraloncology.2015.06.015

6. Gao L, Ren W, Zhang L, Li S, Kong X, Zhang H, et al. PTENpl, a natural sponge of miR-21, mediates PTEN expression to inhibit the proliferation of oral squamous cell carcinoma. Mol Carcinog. 2017 Apr;56(4):1322-34. https://doi.org/10.1002/mc.22594

7. El-Sakka H, Kujan O, Farah CS. Assessing miRNAs profile expression as a risk stratification biomarker in oral potentially malignant disorders: A systematic review. Oral Oncol. 2018 Feb;77:57-82. https://doi.org/10.1016/i.oraloncology.2017.11.021

8. Jia YF, Xiao DJ, Ma XL, Song YY, Hu R, Kong Y, et al. Differentiated embryonic chondrocyte-expressed gene 1 is associated with hypoxia-inducible factor $1 \alpha$ and Ki67 in human gastric cancer. Diagn Pathol. 2013 Feb;8(1):37. https://doi.org/10.1186/1746-1596-8-37

9. Jia $Y, H \cup R$, Li P, Zheng Y, Wang Y, MaX. DECl is required for anti-apoptotic activity of gastric cancer cells under hypoxia by promoting Survivin expression. Gastric Cancer. 2018 Jul;21(4):632-42. https://doi.org/10.1007/s10120-017-0780-z

10. Wang W, Reiser-Erkan C, Michalski CW, Raggi MC, Quan L, Yupei Z, et al. Hypoxia inducible BHLHB2 is a novel and independent prognostic marker in pancreatic ductal adenocarcinoma. Biochem Biophys Res Commun. 2010 Oct;401(3):422-8. https://doi.org/10.1016/j.bbrc.2010.09.070

11. Warnakulasuriya S, Reibel J, Bouquot J, Dabelsteen E. Oral epithelial dysplasia classification systems: predictive value, utility, weaknesses and scope for improvement. J Oral Pathol Med. 2008;37(3):127-33. https://doi: 10.1111/j.1600-0714.2007.00584.x

12. Tan N, Liu Q, Liu X, Gong Z, Zeng Y, Pan G, et al. Low expression of B-cell-associated protein 31 in human primary hepatocellular carcinoma correlates with poor prognosis. Histopathology. 2016 Jan;68(2):221-9. https://doi.org/10.1111/his.12738

13. Cao W, Younis RH, Li J, Chen H, Xia R, Mao L, et al. EZH2 promotes malignant phenotypes and is a predictor of oral cancer development in patients with oral leukoplakia. Cancer Prev Res (Phila). 2011 Nov;4(11):1816-24. https://doi.org/10.1158/1940-6207.CAPR-11-0130

14. Zeidler SV, Botelho TS, Mendonça EF, Batista AC. E-cadherin as a potential biomarker of malignant transformation in oral leukoplakia: a retrospective cohort study. BMC Cancer. 2014 Dec;14(1):972. https://doi.org/10.1186/1471-2407-14-972

15. D'souza B, Nayak R, Kotrashetti VS. Immunohistochemical expression of podoplanin in clinical variants of oral leukoplakia and its correlation with epithelial dysplasia. Appl Immunohistochem Mol Morphol. 2018 Feb;26(2):132-9. https://doi.org/10.1097/pai.0000000000000383

16. Mane DR, Bhat K, Kale AD, Hallikerimath S. Immunoexpression of tenascin as a predictor of the malignancy potential of oral leukoplakia associated with a tobacco habit. Biotech Histochem. 2015;90(7):544-51. https://doi.org/10.3109/10520295.2015.1015055 
17. Waal I. Potentially malignant disorders of the oral and oropharyngeal mucosa; terminology, classification and present concepts of management. Oral Oncol. 2009 Apr-May;45(4-5):317-23. https://doi.org/10.1016/i.oraloncology.2008.05.016

18. Kujan O, Shearston K, Farah CS. The role of hypoxia in oral cancer and potentially malignant disorders: a review. J Oral Pathol Med. 2017 Apr;46(4):246-52. https://doi.org/10.1111/jop.12488

19. Asanoma K, Liu G, Yamane T, Miyanari Y, Takao T, Yagi H, et al. Regulation of the Mechanism of TWISTI Transcription by BHLHE40 and BHLHE41 in Cancer Cells. Mol Cell Biol. 2015 Dec;35(24):4096-109. https://doi.org/10.1128/MCB.00678-15

20. Wang C, Liu W, Liu Z, Chen L, Liu X, Kuang S. Hypoxia inhibits myogenic differentiation through p53 protein-dependent induction of Bhlhe40 protein. J Biol Chem. 2015 Dec;290(50):29707-16. https://doi.org/10.1074/jbc.M115.688671

21. Campo-Trapero J, Cano-Sánchez J, Palacios-Sánchez B, Llamas-Martínez S, Lo Muzio L, Bascones-Martínez A. Cellular senescence in oral cancer and precancer and treatment implications: a review. Acta Oncol. 2008;47(8):1464-74. https://doi.org/10.1080/02841860802183612

22. You JM, Su T, Xia K, Liu Q. [Expression of differentiated embryo-chondrocyte expressed gene I in oral squamous cell carcinoma and its clinical significance]. Shanghai Kou Qiang Yi Xue. 2016 Apr;25(2):217-20. Chinese. 